\title{
RESOLUCIÓN ARBITRAL EXTRA PETITA POSLAUDO: ¿PROTESTAR EN SEDE ARBITRAL 0 JUDICIAL?
}

\author{
Rolando A. Martel Chang* \\ Universidad de Lima, Lima, Perú \\ rmartel@ulima.edu.pe \\ Recibido: 15/11/2020 Aprobado: 22/11/2020 \\ doi: https://doi.org/10.26439/iusetpraxis2021.n052.4961
}

\begin{abstract}
RESUMEN. Quienes litigan en el arbitraje esperan que el tribunal arbitral resuelva el fondo de la controversia, lo que según la ley de la materia (Decreto Legislativo $N .^{\circ}$ 1071) se concretiza con la emisión del laudo. No obstante, la propia ley prevé una serie de solicitudes poslaudo que deben ser resueltas por el mismo tribunal arbitral que emitió el primero. Una de esas solicitudes es la de exclusión del laudo, que procede cuando se ha resuelto un extremo que no ha sido sometido a conocimiento del tribunal o que no es susceptible de arbitraje. Sin embargo, ¿qué ocurre cuando el supuesto de exclusión no se ubica en el laudo, sino en la resolución estimatoria poslaudo que resuelve alguna de las solicitudes? El tema es materia de este trabajo. De hecho, en la casuística judicial sobre anulación de laudos se han presentado demandas bajo este supuesto, que -como se dijo- no tiene regulación específica en la ley de la materia, y por eso consideramos necesario su abordaje, atendiendo a la importancia del arbitraje y sobre todo al derecho a la tutela efectiva que les asiste a los litigantes.
\end{abstract}

PALABRAS CLAVE: arbitraje / laudo arbitral / solicitud de exclusión / resolución poslaudo / recurso de anulación de laudo

\footnotetext{
* Doctor en Derecho por la Universidad de San Martín de Porres. Profesor universitario en la Universidad de San Martín de Porres, en Pontificia Universidad Católica del Perú y en la Universidad de Lima. Juez superior. Presidente de la Corte Superior de Justicia de Lima (2017-2018). Presidente de la Primera Sala Comercial de la Corte Superior de Justicia de Lima. Autor de libros y artículos en materia procesal.
} 


\section{POST-AWARD EXTRA PETITA RESOLUTION: TO PROTEST BEFORE THE ARBITRAL TRIBUNAL OR THE JUDICIAL BRANCH?}

ABSTRACT. Those who litigate in arbitration expect the arbitral tribunal to resolve the merits of the dispute which, according to the law on the subject (Legislative Decree No. 1071), take place with the issuance of the award. However, the law itself provides for a series of post-award requests that must be resolved by the same arbitral tribunal that issued the first award. One of said requests is to exclude the award, which proceeds when an issue that has not been submitted to the court or is not subject to arbitration has been resolved. Nevertheless, what happens when the exclusion assumption is not included in the award but in the post-award resolution that settles any of the requests? The aforementioned is the subject matter of this paper. In fact, in the judicial casuistry on annulment of awards, lawsuits have been filed under this assumption, which-as mentioned before-does not have a specific regulation. For that reason, it is necessary to address this topic, taking into account the importance of arbitration and, overall, the right to effective judicial protection that litigants have.

KEYWORDS: arbitration / arbitration award / request for exclusion / post-award resolution / appeal for annulment of award 


\section{INTRODUCCIÓN}

Desde hace algunos años, el arbitraje, como mecanismo de solución de controversias, tiene en nuestro país un importante desarrollo y uso, no solo en controversias que involucran a personas naturales y jurídicas de la actividad privada, sino también a entidades del sector estatal. La evidencia de lo dicho fluye de la casuística que manejan y administran los centros de arbitraje (Cámara de Comercio de Lima, PUCP, OSCE, entre otros), y de la importante carga procesal que tienen las dos Salas Comerciales de la Corte Superior de Justicia de Lima en materia de recursos de anulación de laudos.

De esta forma, el arbitraje, como mecanismo heterocompositivo de solución de controversias, tiene un gran papel en el rol estatal de brindar a la población tutela efectiva dentro de los procesos arbitrales, donde se decide sobre los derechos sustantivos de las partes que litigan en ellos. La importancia del arbitraje se mantiene a pesar de los hechos de dominio público que han impactado sobre él (como el caso Odebrecht, entre otros), y por ello corresponde, incluso al Poder Judicial en los procesos que conoce, adoptar decisiones que contribuyan a preservarlo.

El arbitraje aparece previsto en nuestra Constitución como jurisdicción. Existe debate, y de hecho así ocurre, sobre la naturaleza jurídica del arbitraje. Entre nosotros, el Tribunal Constitucional considera al arbitraje como jurisdicción. Así se lee, por ejemplo, en el precedente del caso María Julia (STC. Exp. N. ${ }^{\circ}$ 142-2011-AA/TC, 2011).

\section{EL LAUDO ARBITRAL Y EL PRINCIPIO DE CONGRUENCIA PROCESAL}

De acuerdo con el artículo 54 de la Ley de Arbitraje, con la emisión del laudo se decide la controversia. En tal sentido, el laudo arbitral (al igual que la sentencia en el proceso judicial) es el acto procesal más importante del proceso, porque debe contener la decisión de fondo que adopte el tribunal arbitral respecto a la(s) pretensión(es) postulada(s) en la demanda o en la reconvención arbitral. El laudo cierra el debate ocurrido al interior del proceso arbitral.

Pero para que el laudo arbitral tenga ese contenido y fuerza jurídica, es necesario que su dictado se efectúe con pleno respeto del conjunto de garantías mínimas que le asiste a toda parte en el proceso arbitral, pues, de lo contrario, su validez estará seriamente comprometida. Dado que en el proceso arbitral se ventilan derechos patrimoniales o disponibles, dentro de ese conjunto de garantías se ubica el respeto al principio de congruencia procesal', en virtud del cual el tribunal arbitral, al resolver, debe hacerlo

1 El artículo VII del Título Preliminar del Código Procesal Civil se refiere a este principio: "Artículo VII.- El juez debe aplicar el derecho que corresponda al proceso, aunque no haya sido invocado por las partes o lo haya sido erróneamente. Sin embargo, no puede ir más allá del petitorio ni fundar su decisión en hechos diversos de los que han sido alegados por las partes". 
respetando las pretensiones postuladas en la demanda o en la reconvención arbitrales, así como los hechos alegados por las partes. No más que eso, ni menos, ni cosa distinta.

El principio de congruencia "[e]s el principio normativo que exige la identidad jurídica entre lo resuelto, en cualquier sentido, por el juez en la sentencia y las pretensiones y excepciones planteadas por las partes [...]" (Devis Echandía, 1984, p. 49). La falta de adecuación entre la parte dispositiva y las pretensiones deducidas en el proceso admite distintas manifestaciones: que la sentencia otorgue más de lo solicitado por el actor, que conceda menos de lo admitido por el demandado o que resuelva cosa distinta de lo pedido por ambas partes, omitiendo así el pronunciamiento respecto de las pretensiones deducidas en juicio (Picó i Junoi, 1997, p. 66).

De ocurrir lo contrario, esto es, si el tribunal resuelve sin respetar las pretensiones propuestas o sin considerar las alegaciones de las partes, se afecta el principio de congruencia procesal, así como también el derecho de defensa de la parte que no pudo realizar actos de alegación ni de prueba sobre el extremo que ha sido decidido sin haber sido postulado como pretensión. Este exceso es el que da lugar a laudos extra petita.

Devis Echandía (1984) sostiene que el principio de congruencia:

Tiene extraordinaria importancia [...], pues se liga íntimamente con el derecho constitucional de defensa, ya que este exige que el ajusticiado en cualquier clase de proceso conozca las pretensiones o las imputaciones que contra él o frente a él se han formulado, por lo que la violación de la congruencia implica la de aquel derecho; la actividad probatoria, las excepciones o simples defensas y las alegaciones se orientan lógicamente por las pretensiones, imputaciones, excepciones y defensas formuladas en el proceso. (p. 50)

Existe doctrina que entiende que esta causal solo opera cuando el fallo es extra petita, mas no cuando el fallo es ultra petita (Reggiardo Saavedra, 2016, p. 366). En opinión nuestra, en ambos casos existe exceso. En efecto, cuando el fallo es extra petita el tribunal ha resuelto sobre una pretensión no solicitada, y cuando el fallo es ultra petita resuelve en función de las pretensiones solicitadas, pero lo hace excediéndose al otorgar más de lo solicitado. Así, en cualquiera de estos dos escenarios debería proceder la causal de anulación por afectar el principio de congruencia.

Reggiardo Saavedra (2016) señala que "es incorrecto sostener que el fallo ultra petita, donde se otorga más de lo solicitado, sí es válido porque finalmente los árbitros se han pronunciado sobre una materia sometida a su decisión" (p. 367). El autor indica que, independientemente de que los árbitros puedan o no aplicar el principio de iura novit curia, es el principio de congruencia el que limita la aplicación del primero, ya que es este último el que garantiza que no se otorgue algo que no ha sido solicitado por el demandante.

Lo expuesto revela, entonces, que la validez del laudo arbitral tiene como uno de sus límites que se emita respetando plenamente el principio de congruencia procesal. 


\section{LA SOLICITUD DE EXCLUSIÓN DEL LAUDO POR RESOLVER UN EXTREMO NO SOMETIDO A CONOCIMIENTO DEL TRIBUNAL ARBITRAL}

Una vez emitido y notificado el laudo, las partes tienen derecho a presentar, en caso de que corresponda, las solicitudes que están previstas en el artículo 58 del Decreto Legislativo N. ${ }^{\circ}$ 1071, Decreto Legislativo que norma el arbitraje ${ }^{2}$, a saber: de rectificación, de interpretación, de integración y de exclusión.

Para efectos de este trabajo, interesa la solicitud de exclusión, la cual, de acuerdo con la disposición contenida en el inciso d) del numeral 1 del citado artículo 58, se presenta cuando en el laudo:

1. Se ha emitido pronunciamiento sobre algún extremo sin que estuviera sometido a conocimiento y decisión del tribunal arbitral.

2. Que no sea susceptible de arbitraje.

De los dos supuestos antes anotados, el primero es el que se relaciona con el principio de congruencia procesal, en la medida que, si bien se acepta la competencia del

2 Artículo 58.- Rectificación, interpretación, integración y exclusión del laudo.

1. Salvo acuerdo distinto de las partes o disposición diferente del reglamento arbitral aplicable: a. Dentro de los quince (15) días siguientes a la notificación del laudo, cualquiera de las partes puede solicitar la rectificación de cualquier error de cálculo, de trascripción, tipográfico o informático o de naturaleza similar.

b. Dentro de los quince (15) días siguientes a la notificación del laudo, cualquiera de las partes puede solicitar la interpretación de algún extremo oscuro, impreciso o dudoso expresado en la parte decisoria del laudo o que influya en ella para determinar los alcances de la ejecución. c. Dentro de los quince (15) días siguientes a la notificación del laudo, cualquiera de las partes puede solicitar la integración del laudo por haberse omitido resolver cualquier extremo de la controversia sometida a conocimiento y decisión del tribunal arbitral.

d. Dentro de los quince (15) días siguientes a la notificación del laudo, cualquiera de las partes puede solicitar la exclusión del laudo de algún extremo que hubiera sido objeto de pronunciamiento, sin que estuviera sometido a conocimiento y decisión del tribunal arbitral o que no sea susceptible de arbitraje.

e. El tribunal arbitral pondrá la solicitud en conocimiento de la otra parte por quince (15) días. Vencido dicho plazo, con la absolución o sin ella, el tribunal arbitral resolverá la solicitud en un plazo de quince (15) días. Este plazo puede ser ampliado a iniciativa del tribunal arbitral por quince (15) días adicionales.

f. El tribunal arbitral podrá también proceder a iniciativa propia a la rectificación, interpretación o integración del laudo, dentro de los diez (10) días siguientes a la notificación del laudo.

2. La rectificación, interpretación, integración y exclusión formará parte del laudo. Contra esta decisión no procede reconsideración. La notificación de estas decisiones deberá realizarse dentro del plazo pactado por las partes, establecido en el reglamento arbitral aplicable o, en su defecto, en este artículo.

3. Si el tribunal arbitral no se pronuncia acerca de la rectificación, interpretación, integración y exclusión solicitadas dentro del plazo pactado por las partes, establecido en el reglamento arbitral aplicable o, en su defecto, en este artículo, se considerará que la solicitud ha sido denegada. No surtirá efecto cualquier decisión sobre rectificación, interpretación, integración y exclusión del laudo que sea notificada fuera de plazo. 
tribunal para resolver la controversia, lo que se objeta es que se ha laudado en exceso, esto es, sobre un extremo no sometido a conocimiento del tribunal. En cambio, en el segundo supuesto, el tribunal no tiene competencia porque el extremo no es arbitrable, y el laudo mismo es inválido por este motivo, no por afectar la congruencia. Para que exista incongruencia, se requiere de materia arbitrable y que en el laudo se haya resuelto un extremo no sometido a conocimiento del tribunal arbitral.

Ahora bien, en cuanto al trámite, conforme al mismo artículo 58, el tribunal arbitral resuelve previo conocimiento de la parte contraria. Y, si no se pronuncia dentro del plazo pactado por las partes, establecido en el reglamento arbitral aplicable o en dicho artículo, se considerará que la solicitud ha sido denegada.

La solicitud de exclusión permite que el propio tribunal revise si efectivamente el laudo que ha emitido vulnera o no el principio de congruencia procesal por haberse pronunciado sobre una materia que no había sido puesta en su conocimiento. Si el tribunal ampara la solicitud de exclusión, desaparece el defecto y el laudo ya no será impugnado por lo menos por dicho defecto. Por el contrario, si la solicitud es desestimada, es probable que la parte afectada acuda a sede judicial a través del recurso de anulación de laudo. Nótese, pues, la importancia y valía de las solicitudes poslaudo, que, dependiendo de la respuesta del tribunal (resolución estimatoria), podrían evitar la impugnación del laudo.

\section{RESOLUCIONES POSLAUDO Y EL PRINCIPIO DE CONGRUENCIA}

Ya hemos dicho que la tarea arbitral (que incluye la emisión del laudo) debe hacerse respetando el principio de congruencia procesal. Este mismo respeto debe tener el tribunal arbitral al emitir la resolución poslaudo (esto es, a la hora de atender y resolver una solicitud poslaudo), pues, de lo contrario, su validez y eficacia jurídica está en juego.

Acorde con lo que se ha venido anotando, cuando existe incongruencia en el laudo, la parte afectada debe presentar la solicitud de exclusión, que será resuelta por el tribunal. Dicha resolución no será necesariamente estimatoria, pues podría ser desestimatoria.

Ahora bien, la casuística judicial en materia de anulación de laudos está informando de la presentación de recursos de anulación de laudo donde el exceso (haber resuelto sobre un extremo no sometido a conocimiento del tribunal arbitral) no se atribuye al laudo mismo (resolución originaria), sino a la resolución estimatoria de la solicitud poslaudo, la cual pasa a formar parte del laudo, conforme se desprende de las reglas del artículo 63.2 de la Ley de Arbitraje.

La imputación de que el tribunal arbitral ha resuelto en exceso al expedir la resolución poslaudo, es decir que ha resuelto afectando la congruencia procesal, ha sucedido por ejemplo en el Expediente N. ${ }^{\circ}$ 00574-2019-0-1817-SP-CO-01, donde la parte demandante que presentó el recurso de anulación expuso lo siguiente: 
Cabe precisar que, ante lo resuelto en el laudo, tanto LA ENTIDAD como EL CONSORCIO presentaron pedidos de interpretación e integración. El primero de ellos fue declarado infundado, en tanto que el pedido de interpretación de EL CONSORCIO fue declarado fundado en parte y, como consecuencia de ello, se precisó el contenido del laudo en los extremos que se detallan a continuación:

\section{SE RESUELVE:}

1) PRIMERO: Declarar INFUNDADA la solicitud de interpretación e integración formulada por la Municipalidad de San Román.

2) SEGUNDO: Declarar FUNDADA EN PARTE la solicitud de interpretación formulada por el Consorcio Supervisión Juliaca debiéndose precisar que:

a. El pago de las valorizaciones 4, 5, 6, 7, 8 y 9 es más intereses contados a partir de la fecha en que estas valorizaciones debían ser pagadas.

b. Tanto la carta fianza por adelanto como por fiel cumplimiento deben ser devueltas al Consorcio Supervisor Juliaca.

En cuanto a los demás pedidos de integración son estos INFUNDADOS.

3) TERCERO: PRECISAR que la decisión contenida en la presente resolución forma parte del Laudo Arbitral.

- LA ENTIDAD ha presentado la solicitud de interpretación e integración contra el laudo, lo que ha sido declarado infundado, pero contradictoriamente se ha resuelto declarar FUNDADA EN PARTE la solicitud de interpretación e integración formulada por EL CONSORCIO, precisándose que el pago de las valorizaciones es más intereses.

- En base a lo resuelto en relación a tal solicitud post laudo, se ha agregado el pago de intereses, pese a que tal extremo “... NO HA SIDO PRETENDIDO POR EL CONSORCIO EN SU DEMANDA ARBITRAL, ES MÁS ESTA PRETENSIÓN DE PAGO DE INTERESES NO FUE SOMETIDA A ARBITRAJE, mucho menos es PUNTO CONTROVERTIDO, COMO SE ADVIERTE DEL TENOR DE LOS PUNTOS CONTROVERTIDOS EXPUESTOS EN LA PÁG. 19, 20 y 22 del laudo arbitral...".

- Finalmente, señala que al haberse ordenado el pago de valorizaciones más intereses, el tribunal arbitral ha invalidado el laudo, “... porque ha resuelto sobre materias no sometidas a su decisión por las partes...", ordenando el pago de las valorizaciones 4, 5, 6, 7, 8 y 9 más intereses, lo que califica como un pronunciamiento que no se ha basado en actuaciones arbitrales entre las partes en este proceso y no constan como acuerdo entre las partes, de tal forma que LA ENTIDAD no ha podido hacer valer el derecho conforme a ley.

Igual ha sucedido en el Expediente N. $.^{\circ} 00450-2019-0-1817-S P-C 0-01$, donde la parte demandante que presentó el recurso de anulación alega lo siguiente:

Como se menciona, la aclaración efectuada por el Tribunal Arbitral en la resolución $\mathrm{N} .^{\circ} 15$ se da como respuesta al pedido de interpretación presentado por su parte. El Tribunal ha declarado fundado el pedido de interpretación y ha señalado 
que modifica y reemplaza lo resuelto en el primer punto resolutivo de la siguiente manera:

“Primero: Declarar FUNDADA EN PARTE la Primera Pretensión de la demanda. En consecuencia, declara y reconoce que el Contratista tiene el derecho a que se le reconozcan los gastos generales y costos directos derivados de la ampliación de plazo $\mathrm{N} .^{\circ}$, que sean debidamente acreditados, en cualquier momento hasta la liquidación del contrato. Pecto de la suma solicitada, el Tribunal Arbitral concluye que NO CORRESPONDE declarar la suma de 1'677,455.79 por conceptos de gastos generales variables y costos directos, toda vez que el contratista no ha cumplido con acreditar, en el presente arbitraje, la suma antes mencionada".

Al respecto, cabe señalar que, como se puede apreciar en las pretensiones planteadas en la demanda y los puntos controvertidos fijados al interior del proceso arbitral, en ningún momento se solicitó al tribunal arbitral que se declare que el contratista tiene derecho a que se le reconozcan los gastos generales y costos directos derivados de la ampliación de plazo N. ${ }^{\circ}$, que sean debidamente acreditados, en cualquier momento hasta la liquidación del contrato. Lo que únicamente se puso a consideración del tribunal fue que se pronuncie sobre si le corresponde o no al contratista la suma de 1677 455,79 soles por concepto de gastos generales variables y costos fijos. Por eso, es respecto de esto último que debió emitir pronunciamiento y no ir más allá de lo peticionado estableciendo algo que las partes no le solicitaron.

Estos dos casos judiciales dan cuenta precisa del objeto de estudio de este trabajo, pues se acusa que el tribunal arbitral ha resuelto afectando el principio de congruencia a la hora de emitir la resolución estimatoria poslaudo, donde se habría pronunciado sobre un extremo no sometido a su conocimiento.

\section{EL RECURSO DE ANULACIÓN DE LAUDO EXTRA PETITA}

Como es propio de toda demanda, también tratándose del recurso de anulación corresponde al órgano jurisdiccional competente calificar si este cumple o no con los requisitos de admisibilidad y de procedibilidad que establece la ley.

Con relación al objeto de estudio de este trabajo, el artículo 63.2 del Decreto Legislativo N. ${ }^{\circ}$ 1071, Decreto Legislativo que norma el arbitraje, establece lo siguiente:

Artículo 63.- Causales de anulación

$[\ldots]$

2. Las causales previstas en los incisos a, b, c y d del numeral 1 de este artículo solo serán procedentes si fueron objeto de reclamo expreso en su momento ante el tribunal arbitral por la parte afectada y fueron desestimadas. 
Según la citada disposición, cuando se presenta un recurso de anulación de laudo arbitral donde se invoca la causal del inciso d) del numeral 1 del artículo 63 de la Ley de Arbitraje, esto es, por haberse emitido un laudo extra petita, el órgano jurisdiccional debe verificar si el demandante ha cumplido con presentar la solicitud de exclusión y si esta ha sido desestimada, pues de lo contrario el recurso es improcedente.

La disposición en comentario contempla la posibilidad de presentar la solicitud de exclusión respecto del laudo, a fin de que el propio tribunal solucione, de ser el caso, el defecto del laudo por exceso.

Lo que no se ha contemplado en esa disposición ni en ninguna otra de la Ley de Arbitraje es si debe procederse de igual manera cuando el exceso no está en el laudo mismo, sino en la resolución estimatoria que resuelve alguna solicitud poslaudo (como podría ser de integración o de interpretación) y que pasa a formar parte del laudo.

Los casos judiciales de anulación de laudo arbitral mencionados en este trabajo dan cuenta de la situación antes descrita, frente a la cual la respuesta jurisdiccional ha sido favorecer el acceso a la justicia con base en el principio pro actione. A este criterio puede sumarse con suma facilidad el argumento de que la disposición del artículo 63.2 es de carácter restrictivo, en la medida que impone una barrera para quien decida acudir a sede judicial para anular un laudo arbitral, barrera que solo debe operar cuando el supuesto de improcedencia es claro y expreso, mas no cuando ello no suceda.

Esto es justamente lo que se ha encontrado en la situación que aborda este trabajo, donde la vulneración al principio de congruencia recién aparece en la resolución estimatoria poslaudo. En efecto, si la disposición que prevé un requisito de procedibilidad de la demanda es de carácter restrictivo, no puede por su propia naturaleza ser interpretada en sentido expansivo, lo que sí ocurre con disposiciones favorables a los derechos de los litigantes.

En el Expediente N.00574-2019-0-1817-SP-C0-01, la Sala Comercial argumenta lo siguiente:

Décimo Quinto. Ahora bien, en relación a la causal de anulación basada en el literal "d" del numeral 63.1 del Decreto Legislativo 1071, lo primero que debemos destacar es que el pago de intereses que da lugar al pedido de anulación de LA ENTIDAD es una decisión que recién aparece como consecuencia de la emisión de la resolución post laudo del 30 de septiembre de 2019, y LA ENTIDAD optó por acudir directamente al Poder Judicial sin realizar reclamo alguno ante el Tribunal Arbitral. En tal contexto, en el particular caso que ahora se analiza, no corresponde exigir a LA ENTIDAD que haya hecho uso del reclamo previo que prevé el numeral 63.2 del Decreto Legislativo 1071, pues nos encontramos ante una circunstancia no prevista en tal enunciado normativo, por lo que en observancia del principio pro actione corresponde analizar el aspecto sustantivo de tal cuestionamiento. 
Y, en el Expediente N. ${ }^{\circ}$ 00450-2019-0-1817-SP-CO-01, este mismo órgano jurisdiccional argumenta lo siguiente:

SEXTO: Comparando los argumentos de la citada solicitud de interpretación del laudo arbitral, con los argumentos referidos en el recurso de anulación de laudo presentado sobre la causal b) de la Ley de Arbitraje, se verifica con claridad que los temas propuestos en el presente recurso de anulación han sido postulados también en el citado recurso post laudo, razón por la cual corresponde a este Colegiado Superior emitir pronunciamiento en torno a la anulación presentada por el recurrente. Asimismo, respecto a la causal d), estando a que el pedido de exclusión se ha realizado contra la resolución que resuelve el recurso post laudo, corresponde emitir pronunciamiento en torno a ello, toda vez que la ley arbitral no prevé solicitud alguna contra esta resolución post laudo.

Al margen del resultado de los dos casos judiciales antes señalados, que no fue favorable a la parte reclamante, lo cierto es que la protesta da cuenta de una situación concreta no prevista en la ley de la materia, frente a la cual el órgano jurisdiccional ha optado por favorecer los derechos constitucionales de acceso a la justicia y a la tutela efectiva, pero que bien podría dar lugar a una reforma legal que, regulando esta situación, permita a la parte afectada formular su reclamo en sede arbitral y al tribunal resolver (y en su caso, corregir) el exceso que pudiera existir en el laudo complementado con la resolución poslaudo. De este modo, será siempre el tribunal arbitral quien tendrá la posibilidad legal de superar, si fuera el caso, el defecto del laudo por exceso. Como están las cosas ahora, tal posibilidad solo la tiene cuando el exceso está en el laudo, mas no cuando está en la resolución estimatoria poslaudo, que, como se dijo, forma parte del laudo.

Claro que, en tanto no haya regulación legal específica, consideramos que la solución que ha adoptado el órgano jurisdiccional es conforme al ordenamiento jurídico y a los derechos constitucionales antes mencionados.

\section{EL RECURSO DE ANULACIÓN DE LAUDO POR AFECTARSE LA CONGRUENCIA CON BASE EN LAS CAUSALES B) Y C) DEL ARTíCULO 63, NUMERAL 1, DE LA LEY DE ARBITRAJE}

Bajo la idea de que el laudo ha afectado el principio de congruencia, en sede judicial se han presentado recursos de anulación acusando que con ese defecto se ha vulnerado el principio de motivación de las resoluciones. La casuística muestra que esta protesta se ha encuadrado dentro de las causales previstas en los incisos b) y c) del numeral 1 del artículo 63 de la Ley de Arbitraje, a saber:

Artículo 63.- Causales de anulación

1. El laudo solo podrá ser anulado cuando la parte que solicita la anulación alegue y pruebe: 
[...]

b. Que una de las partes no ha sido debidamente notificada del nombramiento de un árbitro o de las actuaciones arbitrales, o no ha podido por cualquier otra razón hacer valer sus derechos [cursivas añadidas].

c. Que la composición del tribunal arbitral o las actuaciones arbitrales no se han ajustado al acuerdo entre las partes 0 al reglamento arbitral aplicable [cursivas añadidas], salvo que dicho acuerdo o disposición estuvieran en conflicto con una disposición de este Decreto Legislativo de la que las partes no pudieran apartarse, o en defecto de dicho acuerdo o reglamento, que no se han ajustado a lo establecido en este Decreto Legislativo.

[...]

Respecto al contenido esencial del derecho a la motivación, el Tribunal Constitucional expresa lo siguiente:

El derecho a la debida motivación de las resoluciones judiciales se respeta siempre que exista fundamentación jurídica, congruencia entre lo pedido y lo resuelto [cursivas añadidas] y por sí mismo exprese una suficiente justificación de la decisión adoptada, aun si esta es breve o concisa, o se presenta un supuesto de motivación por remisión. (STC. Exp. N. ${ }^{\circ} 04228-2005-\mathrm{HC} / T C, 2006$, fundamento jurídico 1)

Lo anotado por el Tribunal Constitucional deja claro que, cuando se afecta el principio de congruencia, también se afecta la motivación de las resoluciones.

Tratándose de este defecto de motivación de las resoluciones, en la praxis judicial se ha establecido que ninguna de las solicitudes poslaudo permite que el propio tribunal arbitral subsane los defectos de motivación que acusa la parte reclamante, y desde este punto de vista no es necesario que se presente en sede arbitral solicitud alguna. Ahora bien, si el interesado decide presentarla, tal hecho no afecta su derecho de acudir a la anulación del laudo ${ }^{3}$.

\section{CONCLUSIONES}

La realidad del litigio, arbitral y judicial, siempre supera cualquier programa legal, en la medida que los casos concretos dan cuenta de situaciones no previstas en dicho programa.

Los requisitos de procedibilidad de toda demanda son verificables por los órganos jurisdiccionales, en la medida que su exigencia esté clara y expresamente prevista en una disposición legal. Esta disposición es, entonces, restrictiva del derecho de acceso a la justicia, y si no existe esa disposición expresa, debe favorecerse el acceso a la justicia.

3 Puede consultarse mayor explicación sobre este tema en Martel (2018, pp. 34-40). 
Si bien las situaciones no previstas en un programa legal, como lo es la Ley de Arbitraje, se atienden y resuelven acudiendo al ordenamiento jurídico en su conjunto, lo mejor sería que tales situaciones se regulen de modo específico en la ley de la materia, a fin de conservar la idea que contiene el programa legal, como es la de permitir al propio tribunal arbitral la posibilidad de solucionar los problemas de un laudo extra petita, cosa que por ahora no sucede en la situación descrita en este trabajo.

En tanto no haya regulación específica en la ley de la materia que atienda la situación descrita en este trabajo, resulta adecuada la respuesta jurisdiccional de favorecer la protesta de los litigantes que acusan que el laudo ha emitido un pronunciamiento extra petita en la resolución estimatoria poslaudo, lo que debe entenderse en el sentido de dar lugar a la revisión de la protesta, mas no de resolver favorablemente a ella.

\section{REFERENCIAS}

Devis Echandía, H. (1984). Teoría general del proceso. Buenos Aires: Editorial Universidad.

Martel, R. (2018). La anulación de laudos arbitrales en la jurisprudencia. Lima: Gaceta Jurídica.

Picó i Junoy, J. (1997). Las garantías constitucionales del proceso. Barcelona. J. M. Bosch Editor.

Reggiardo Saavedra, M. (2016). La anulación de laudo por defectos de motivación en Perú. En G. Priori Posada (Coord.), Argumentación jurídica y motivación de las resoluciones judiciales (pp. 357-372). Lima: Palestra Editores.

Sentencia recaída en el Expediente N. ${ }^{\circ}$ 04228-2005-HC/TC. (2006). Tribunal Constitucional del Perú. Recuperado de https://www.tc.gob.pe/jurisprudencia/2006/042282005-HC.html

Sentencia recaída en el Expediente N. ${ }^{\circ}$ 00142-2011-PA/TC. (2011). Tribunal Constitucional del Perú. Recuperado de https://www.tc.gob.pe/jurisprudencia/2011/001422011-AA.html 